\title{
An adaptive L2 cache prefetching mechanism for effective exploitation of abundant memory bandwidth of 3-D IC technology
}

\author{
Hong-Yeol Lim ${ }^{\mathrm{a})}$ and Gi-Ho Park ${ }^{\mathrm{b})}$ \\ Department of Computer Engineering, Sejong University, \\ 98, Gunja-Dong, Gwangjin-Gu, Seoul, 143-747, Republic of Korea \\ a)hylim@sju.ac.kr \\ b) ghpark@sejong.ac.kr,correspondingauthor
}

\begin{abstract}
Three-dimensional integration circuits (3-D ICs) technology provides various opportunities for new computer system architectures. Data prefetching, which can utilize the abundant memory bandwidth of 3-D IC technology is one of the most promising approaches in 3D IC technology today. This paper analyzes the efficiency of aggressive prefetching based on the huge bandwidth of 3-D IC technology. The performance analysis results reveal that these simple aggressive prefetching mechanisms cannot achieve performance improvements even with the enormous memory bandwidth of 3-D IC technology. An adaptive L2 cache prefetching mechanism is proposed, which adjusts the amount of prefetching data adaptively based on the memory access pattern to exploit the abundant memory bandwidth effectively. The proposed mechanism can achieve a performance improvement of about $5 \%$ more on average than a conventional $1 \mathrm{MB}$ L2 cache, and it can significantly reduce energy consumption of an L2 cache by about $29 \%$ on average over the conventional $2 \mathrm{MB}$ L2.
\end{abstract}

Keywords: cache prefetching, 3-D ICs technology, L2 cache Classification: Integrated circuits

\section{References}

[1] Samsung Exynos 5, http://www.samsung.com/global/business /semiconductor/product/application/detail?productId $=7668$

[2] N. P. Jouppi: ISCA-17 (1990) 364.

[3] S. Palacharla and R. E. Kessler: ISCA-21 (1994) 24.

[4] K. Inoue, K. Kai and K. Murakami: HPCA-5 (1999) 218.

[5] T. Ono, K. Inoue and K. Murakami: ISOCC 2009 (2009) 472. 
[7] C. Liu, I. Ganusov, M. Burtscher and T. Sandlip: Design \& Test Computers (2005) 556 .

[8] J. Sharkey, D. Ponomarev and K. Ghose: Technical Report CS-TR-05DP01, Department of Computer Science, State University of New York at Binghamton (2005).

[9] A. Jeleel: "Memory Characterization of Workloads Using Instrumentation-Driven Simulation-A Pin-Based Memory Characterization of the SPEC CPU 2000 SPEC 2006 Benchmark Suites", Intel VSSAD Technical Report, 2007.

[10] M. R. Guthaus, J. S. Ringenberg, D. Ernst, T. M. Austin, T. Mudge and R. B. Brown: Workload Characterization (2001) 3.

[11] S. Thoziyoor: "CACTI 5.3", HP Laboratories, Palo Alto, CA, 2008.

\section{Introduction}

Three-dimensional integration circuits (3-D ICs) technology provides various opportunities to devise new computer system architecture through the faster and abundant memory bandwidth much more than conventional 2-D system structures. In addition, the L2 cache of the core becomes larger in capacity to support memory intensive applications such as high-end videos and 3D games etc. [1]. The most conventional approaches to exploit abundant memory bandwidth provided by 3-D IC technology are to increase the cache block size and to perform prefetching from a lower level memory.

However, these approaches can degrade performance easily because of cache pollution and generating too much memory traffic. To overcome these shortcomings, this paper proposes an adaptive L2 cache prefetching mechanism to utilize the enormous memory bandwidth based on 3-D IC technology. The proposed L2 cache prefetching mechanism adjusts the prefetch amount based on the memory access pattern. The performance evaluation results show that the proposed adaptive L2 cache prefetching mechanism with the $512 \mathrm{~KB}$ cache can achieve a performance improvement of about $5 \%$ and $2 \%$ over the conventional $1 \mathrm{MB}$ and $2 \mathrm{MB}$ L2 cache, respectively. In addition, the proposed mechanism can reduce energy consumption by about $29 \%$ on average over the conventional 2 MB L2 cache.

This paper is organized as follows: Section 2 explains related works. The proposed adaptive L2 cache prefetching mechanism and system operational model is described in Section 3. The performance evaluation results are presented in Section 4. Finally, the conclusion is in Section 5.

\section{Related work}

Various mechanisms have been proposed to exploit memory bandwidth efficiently. The stream buffer, as a fully-associative buffer below the L1 directmapped cache is proposed in [2]. Palacharlar et al. extended the idea of the original stream buffer into the L2 cache to reduce long memory access latency in [3]. Adaptive prefetching mechanisms have been investigated as well. Inoue et al., proposed a mechanism that adjusts fetch size based on 
the access pattern of a cache block in [4]. Ono et al. [5] recently proposed a software-controllable mechanism to adjust fetch size dynamically based on the profiling information gathered during the compile time for the 3-D integration technology. In [6] Woo et al., proposed a SMART-3D architecture, which efficiently utilized the TSVs to reduce long latency of fetching and write-back in an L2 cache. Liu et al. [7], investigated various conventional schemes including a stream buffer to bridging the processor and memory performance gap based on the 3-D integration technology. This paper proposes an adaptive L2 cache prefetching mechanism that adjusts the prefetching amount if the previously generated prefetch request is useful.

\section{Adaptive L2 cache prefetching mechanism}

The proposed L2 cache prefetching mechanism is presented in Fig. 1. A cache memory system that has a $32 \mathrm{~KB} 4$-way L1 data cache and a $512 \mathrm{~KB}$ 8-way L2 cache is assumed as a base cache memory system. The line size of L1 is $64 \mathrm{~B}$ and the line size of L2 is $128 \mathrm{~B}$. To perform adaptive L2 cache prefetching, a prefetch bit is equipped with a cache block to indicate whether the cache block is a prefetched block or not. The proposed prefetch mechanism also uses a prefetch reference table (PRT) to store cache block addresses where the prefetch requests were generated by previous cache misses. The table has 64-entries with 32 bits.

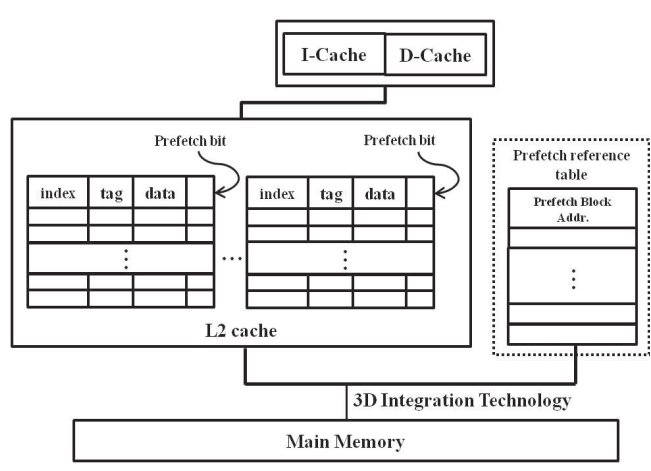

Fig. 1. The system architecture of L2 cache prefetching

The operation model of the proposed L2 cache prefetching mechanism is shown in Algorithm 1. If a cache miss occurs in the L1 and L2 cache, five cache blocks are fetched, including the cache block that contains the data that causes the cache miss. So, the proposed mechanism prefetches four sequential cache blocks next to the missed block. The prefetch bits of these four blocks are set. The address of the first prefetched block is stored in a prefetch reference table as well. If there is a cache hit in the L2 cache, the prefetch bit is checked in regard to whether the cache block is a prefetched cache block or not. If it is a prefetched block, the prefetch reference table is referred with the prefetch block address. If there is a match in the prefetch reference table, a prefetch request is generated for four cache blocks starting from the cache block with a block address of $4^{*}$ block size + block address 
that is matched in the prefetch reference table. The access latency of the prefetch reference table is assumed to be three cycles. The entry of the prefetch reference table is updated as the first prefetched block address, i.e., the current block address $+4 *$ block size. If there is no match in the prefetch reference table, any prefetch request is not generated. The prefetch reference table is accessed using an index scheme based on modulo operation of the block address by the number of entries in the prefetch reference table as a direct-mapped scheme in the cache.

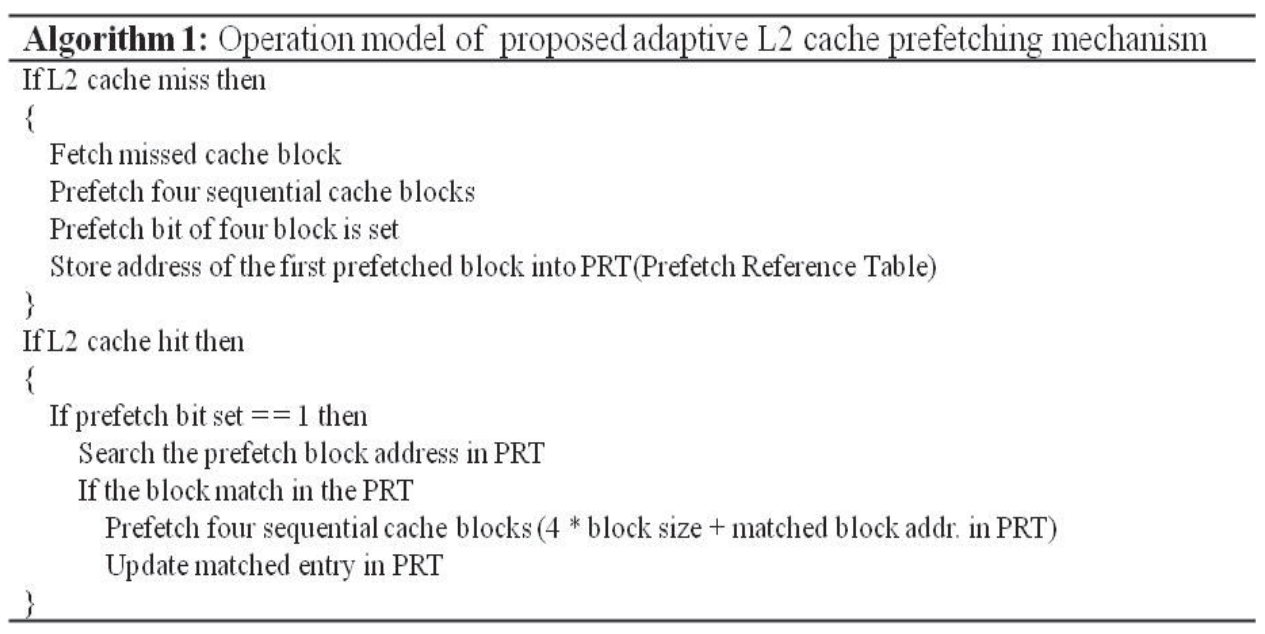

\section{Performance evaluation results}

The system parameters for the simulation are shown in Table I. A performance evaluation was performed based on M-sim simulator [8] with SPEC CPU 2000 [9] and Mibench [10] benchmarks. The access latency and power consumption of memory systems are obtained by the CACTI 5.3 tool [11].

Table I. System parameters

\begin{tabular}{|l|l|}
\hline L1 Cache (Instruction/Data Cache) & $\begin{array}{l}\text { Capacity : 16 KB/16 KB (I/D), Block size : 64 B, } \\
\text { Associativity : 4-way, Hit latency : } 2 \text { cycle }\end{array}$ \\
\hline L2 Cache (Unified Cache) & Capacity : $256 \mathrm{~KB} \sim 2 \mathrm{MB}$, Block size : $64 \mathrm{~B} \sim 1 \mathrm{~KB}$, \\
& Associativity : 8-way, Hit latency : 10 67 \\
\hline Main Memory & Latency (first latency - next chunk latency) : $54-2$, \\
& Memory bandwidth : 512 bit \\
\hline Prefetch reference table & $512 \mathrm{~B}(32$ bit $* 64 \mathrm{entries})$ \\
\hline
\end{tabular}

Firstly, the effect of the block size of L2 is analyzed in the simulation with the high memory bandwidth provided by 3D IC technology. Fig. 2 presents the performance of the $\mathrm{L} 2$ cache with a capacity of $512 \mathrm{~KB}$ having various cache block sizes from $64 \mathrm{~B}$ to $1 \mathrm{~KB}$. As shown in Fig. 2, the performance evaluation results show that the best performance can be achieved when the L2 cache has a $128 \mathrm{~B}$ block size even with an enormous memory bandwidth. 


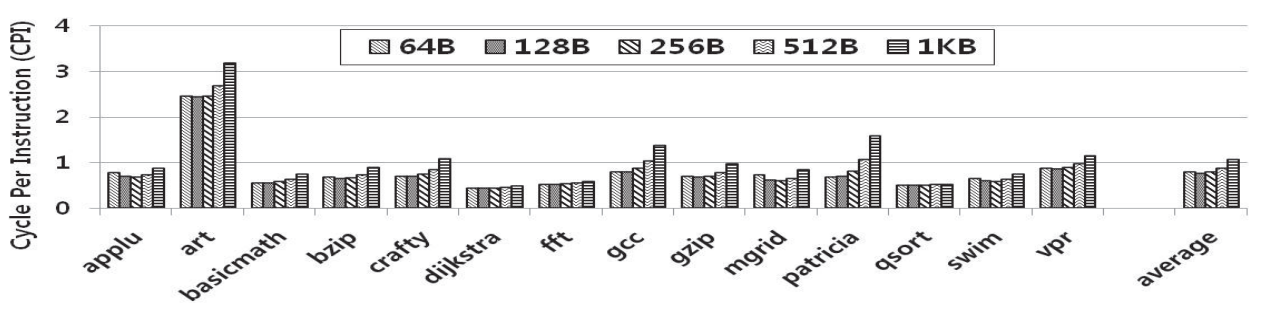

Fig. 2. The performance according to the cache block size of L2 cache

This result shows that simply increasing the block size cannot deliver better performance due to the cache pollution even though the system provides a massive memory access bandwidth. The simulation results show that a simple approach to increase the cache block size is not an appropriate method to exploit the abundant memory bandwidth provided by the 3D IC technology.

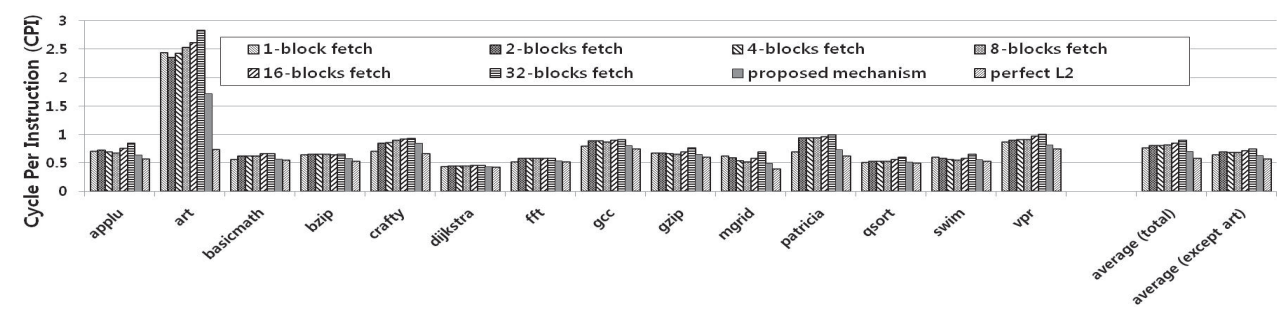

Fig. 3. The performance of the proposed mechanism with various fetch sizes

Fig. 3 shows the performance of the proposed mechanism with those of the conventional cache systems with various fetch sizes. As shown in Fig. 3, the performance of the L2 cache with simple aggressive prefetching that prefetches more cache blocks decreases significantly by about $11 \%$ on average as it increases the fetch block size from 2-blocks (256 B) to 32-blocks ( $4 \mathrm{~KB})$. Note that one-block fetch is the same mechanism to a baseline L2 cache with a capacity of $512 \mathrm{~KB}$ having a $128 \mathrm{~B}$ block size. However, the proposed mechanism can achieve a performance improvement of about $6 \%$ and $12 \%$ on average over the conventional L2 cache fetching 4-blocks (512 B) and 32-blocks (4KB), respectively. Fig. 3, 4 and 5 present the average values that exclude the result of the art benchmark in addition to the overall average value for all benchmarks. This is mainly because the absolute value of CPI, energy consumption of the art benchmark is much higher than the other benchmarks and it may distort the overall results. The results show that those two averages show a similar tendency for the proposed mechanism even though the absolute numbers of the two average values are different.

Fig. 4 presents the performance of the proposed mechanism compared with the conventional cache systems with various capacities. As shown in Fig. 4, the proposed mechanism can achieve a performance improvement of about $7 \%$ over the conventional $512 \mathrm{~KB}$ L2 cache by adaptively prefetching four additional cache blocks (512 B). In addition, the proposed mechanism 


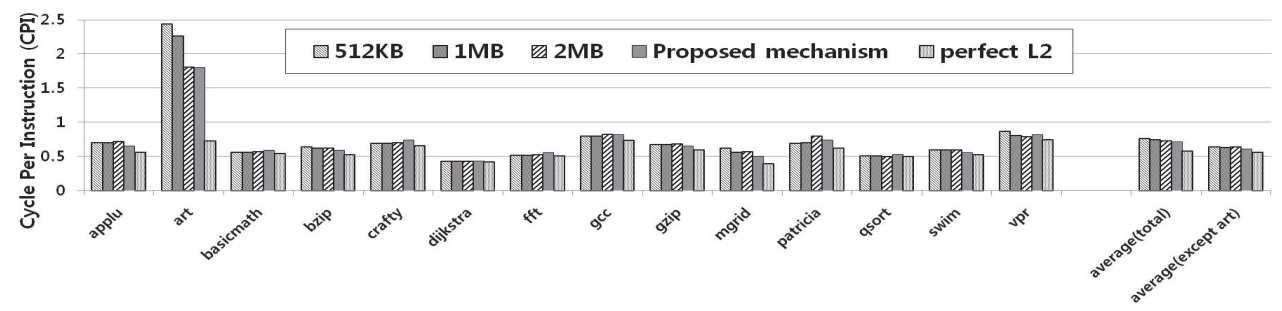

Fig. 4. The performance comparison with a conventional cache with various capacities

can achieve a performance improvement of about $5 \%$ and $2 \%$ over the conventional $1 \mathrm{MB}$ and $2 \mathrm{MB}$ L2 caches having $128 \mathrm{~B}$ block size respectively. This result shows that the proposed mechanism can improve performance significantly by adjusting amount of fetch size based on the memory access pattern. In addition, it shows that a sophisticated prefetching scheme is very important in exploiting the massive memory bandwidth of 3-D IC technology effectively.

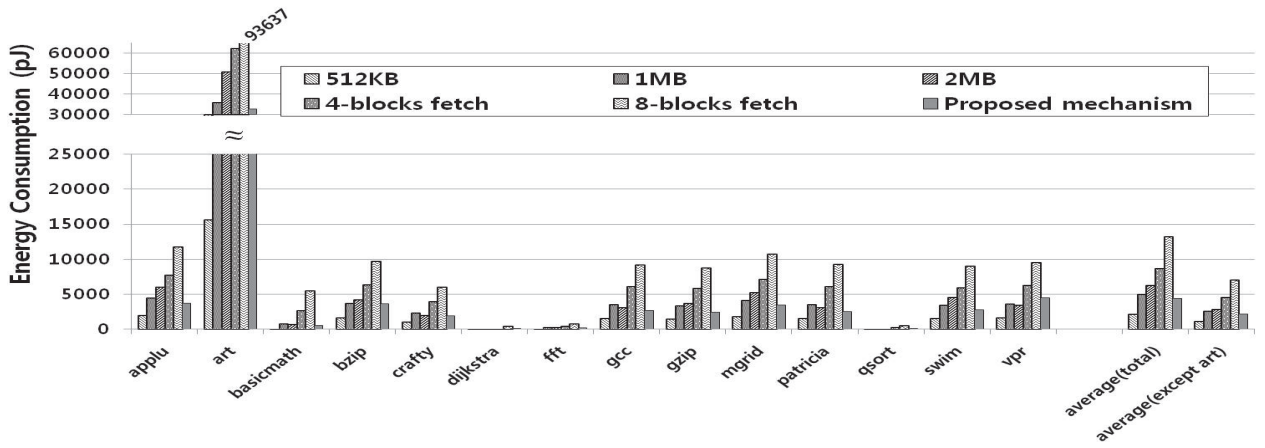

Fig. 5. The energy consumption of proposed mechanism

Fig. 5 shows the energy consumption of an L2 cache in various configurations. The energy consumption of L2 cache hierarchy was measured based on the value from CACTI 5.3 [11]. The energy consumption due to the additional hardware for the proposed mechanism was also considered in this experiment. The major additional hardware components used to perform the proposed mechanism are a PRT and an address incrementer used to generate a prefetch block address. The PRT has a $256 \mathrm{~B}$ capacity (the size of each entry is 32 bit and the number of an entry is 64) and accessing the PRT is the main additional power consumption for the proposed mechanism. To consider the power consumption of additional hardware logic for the proposed mechanism, the power consumption value of a $512 \mathrm{~B}$ PRT, which is two times bigger than an actual PRT, is used for the energy consumption analysis shown in Figure 5. As shown in Fig. 5, the proposed mechanism can reduce the energy dissipation by about $11 \%$ and $29 \%$ on average over the conventional cache with $1 \mathrm{MB}$ and $2 \mathrm{MB}$ capacities, respectively. In addition, it can significantly reduce energy consumption by about $53 \%$ and $68 \%$ 
and 8-blocks, respectively. These results show that the proposed mechanism can significantly reduce energy consumption in addition to the performance improvement over the conventional cache system with a large capacity by prefetching adaptively based on the memory access pattern.

\section{Conclusion}

This paper analyzes the efficiency of simple aggressive prefetching, such as increasing block sizes and prefetching multiple cache blocks based on the huge bandwidth of 3-D IC technology. The performance analysis results reveal that these simple approaches cannot achieve performance improvements even with the enormous memory bandwidth of 3-D IC technology. An adaptive L2 cache prefetching mechanism is also proposed that adjusts the amount of the prefetching data adaptively based on the memory access pattern to exploit the abundant memory bandwidth effectively. The performance evaluation results show that the proposed adaptive L2 cache with a capacity of $512 \mathrm{~KB}$ can achieve a performance improvement of about $5 \%$ and $2 \%$ more than conventional $1 \mathrm{MB}$ and $2 \mathrm{MB}$ L2 caches respectively. The proposed mechanism can reduce energy dissipation by about $29 \%$ more on average than a conventional $2 \mathrm{MB}$ L2 as well. The analysis of the energy efficiency of the prefetching mechanism and more sophisticated adaptive schemes in multi-core environments will be a topic for future research.

\section{Acknowledgments}

This work was supported by the National Research Foundation of Korea (NRF) grant funded by the Korea government (MEST) (No.2010-0006785). 